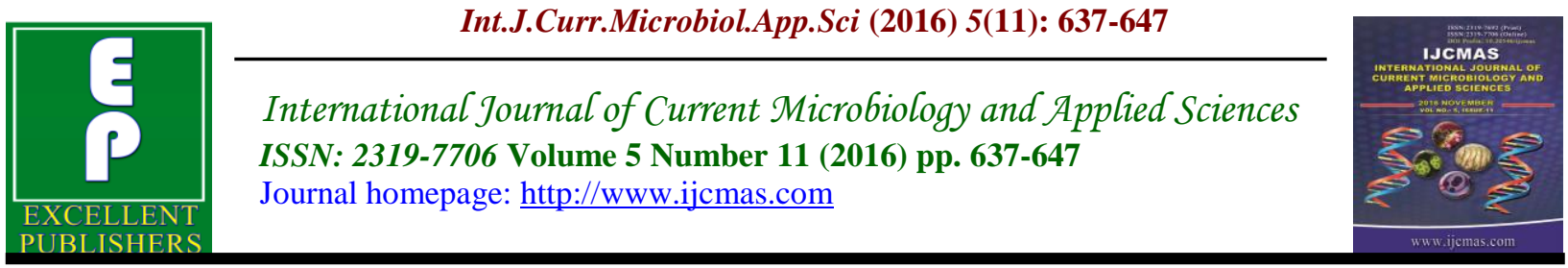

Original Research Article

http://dx.doi.org/10.20546/ijcmas.2016.511.075

\title{
Molecular Characterization of Carbapenem Resistant Acinetobacter baumannii in Cancer Patients
}

\author{
Rasha M. Abdel Hamid ${ }^{1}$, Safaa S. Hassan ${ }^{1}$, Hadir A. El-Mahallawy ${ }^{1}$ and Magdy Saber ${ }^{2}$ \\ ${ }^{1}$ Clinical pathology department, National Cancer Institute, Cairo University, Egypt \\ ${ }^{2}$ Medical Oncology Department, National Cancer Institute, Cairo University, Egypt \\ *Corresponding author
}

Keywords

Multidrug resistance

Acinetobacter

baumannii-

carbapenem

resistance- Metallo-

$\beta$-lactamase

producers- OXA-

type carbapenamases.

Article Info

Accepted:

26 October 2016

Available Online:

10 November 2016

\section{A B S T R A C T}

This study aimed to investigate the prevalence of Multidrug resistant Acinetobacter baumannii (MDR-AB) as a cause of health-care associated infection (HAI) in cancer patients and study the mechanisms of carbapenem resistance in relation to different risk factors. In this study, Multidrug resistant Acinetobacter were isolated and subjected to Imipenem-EDTA combined disk synergy (CDST-IPM), multiplex PCR to detect four families of OXA-type carbapenamases and sequencing of bla $_{\text {OXA-5I }}$ like gene. During one and a half year, MDR-AB accounted for $15 \%$ of gram negative pathogens. The majority of MDR-AB infections were in patients who spent 7 or more days in the hospital $(76.8 \%)$. More than one mechanism of resistance were detected, as metallo- $\beta$-lactamase (MBL) producers, bla OXA-5l $_{\text {like }}$ and bla $_{O X-23}$ like were detected in $80 \%, 94 \%$ and $63 \%$ of MDR-AB; respectively. The bla $_{O X A-23}$ and MBLs coexisted simultaneously in $71 \%$ of cases $(p=0.003)$; and $7 \%$ of imipenem resistant isolates were negative for MBL and bla $a_{O X A-23}$ suggesting other resistance mechanisms. The presence of Acinetobacter resistant genes ( $b l a_{O X A-51}$ and $b l a_{\text {OXA-23 }}$ ) was significantly associated with carbapenem use, $p=0.015$ and 0.064; respectively. The phylogenetic analysis showed different genetic variants. In conclusion, acquisition of carbapenem resistance among MDR-AB could be related to carbapenem therapy and prolonged duration of hospitalization.

\section{Introduction}

Hospitals worldwide are facing a crisis in the upsurge and dissemination of antimicrobial resistant bacteria. Nowadays, MDR-AB infections are increasing leading to limited therapeutic options, and high rates of morbidity and mortality (Fattouh and Nasr El-din, 2014). The evolution of MDR is relatively fast, as the main driving force is lateral gene transfer, via a wide range of mobile genetic elements. Integrons have often been found in plasmids and/or transposons that promote the spread of resistance genes (Prata-Rocha et al., 2013).

Carbapenem resistance is mediated by a combination of different mechanisms. The most commonly encountered mechanisms in A. baumannii are the production of MBLs and carbapenem hydrolyzing class $\mathrm{D}$ betalactamases (CHDLs) (Lee et al., 2013). The production of MBLs is one of the most worrisome resistance mechanisms because they not only limit treatment options but 
also their genes are carried on highly mobile elements, allowing their easy dissemination (Mohamed and Rafaat, 2011). Thus, detection of MBLs may help to modify therapy and necessitate the initiation of effective infection control to prevent further dissemination (Fattouh and Nasr El-din, 2014). Five main subgroups of CHDLs have been recognized in $A$. baumannii, bla $a_{O X A-23}$ like, bla $a_{O X A-40}$ like (originally called bla ${ }_{O X A}$ 24 like), bla ${ }_{O X A-51}$ like, bla $a_{O X A-58}$ like and bla $a_{O X A-143}$ like. The bla $a_{O X A-51}$ like is intrinsic to $A$. baumannii, while the others are acquired (Opazo et al., 2012). The distribution of $A$. baumannii carrying these different acquired CHDL genes varies among different regions and even in different hospitals (Lee et al., 2013). Although CHDLs have a lower catalytic efficiency to hydrolyze carbapenems compared to MBLs, it is important to consider them as potentially dangerous because their expression can be regulated by the upstream insertion of insertion sequence (IS) elements such as ISAba1. This can be intensified in the presence of other resistance mechanisms, such as increased expression of efflux pumps and loss of porins (Opazo et al., 2012).

It has been reported that CHDL-producing carbapenem resistant $A$. baumannii (CRAB) provide a sheltering effect for carbapenem susceptible pathogens and can exacerbate the pathogenesis of polymicrobial infections in case of carbapenem treatment. This indicate a greater importance for the presence of CRAB in clinical settings and emphasize the urgent need for more effective controls of CRAB in polymicrobial infection (Liao et al., 2014).

Thus we aimed to investigate the prevalence of MDR-AB as a cause of HAI in cancer patients and study mechanisms of carbapenem resistance in these pathogens in relation to different risk factors including duration of hospitalization, surgical procedures, empirical carbapenem therapy and ICU admission.

\section{Patients and Methods}

This was a retrospective study conducted at the Microbiology unit of Clinical Pathology Department at National Cancer Institute, Cairo University, Egypt, during the period from January 2012 to May 2013. Patients included in the study were adult ( $\geq 18$ years) cancer patients receiving their treatment in different departments of NCI. The study was approved by the ethical committee review board of NCI, Cairo University and patients' consents were obtained.

Data collection: Medical records of the patients infected with MDR Acinetobacter species were reviewed for the following data including age, gender, the underlying disease, department (medical or surgical), ICU admission, prior hospitalization, duration of hospital stay before onset of infection, clinical site of infection, surgical procedures, chemotherapy, antibiotic received especially carbapenems, duration and clinical outcome of the episode. The isolate was considered as MDR, if resistant to more than two classes of antimicrobials ( $\geq 3$ classes) (Vaze et al., 2013). Carbapenem resistant Acinetobacter isolates were defined as non-susceptible or with diminished susceptibility to meropenem and/or imipenem in vitro (Kim et al., 2012). Fourteen-day in hospital mortality after the onset of Acinetobacter infection was used as the main assessment of patients' outcome.

Microbiology: Non-duplicate MDR Acinetobacter organisms isolated from various clinical samples of cancer patients during the study period were collected and purity colonies were done. The isolates were 
identified using Microscan Walk Away 96, Gram-negative panels. The antibiotic panel included were amikacin, amoxicillinclavulanic acid, aztreonam, cefepime, cefotaxime, cefoxitin, ceftazidime, ceftriaxone, ciprofloxacin, gentamicin, imipenem, levofloxacin, meropenem, piperacillin-tazobactam, tobramycin and trimethoprim-sulfamethoxazole. Results were interpreted according to Clinical and Laboratory Standards Institute (CLSI) guidelines (CLSI, 2010).

Imipenem - EDTA combined disk synergy test (CDST-IPM): Test organisms were inoculated on to Mueller Hinton agar plates.

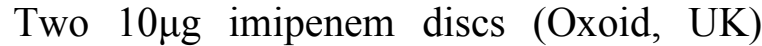
were placed on the plate, and appropriate amounts of $10 \mu 1$ of EDTA solution $(0.5 \mathrm{M})$ was added to one disc. The inhibition zones of the imipenem and imipenem-EDTA discs were compared after 24 hours of incubation at $37^{\circ} \mathrm{C}$. The difference of $\geq 7 \mathrm{~mm}$ between the inhibition zone diameter of the imipenem -EDTA disk and that of imipenem disk alone was considered to be positive for the presence of MBLs (Hodiwala et al., 2013).

Multiplex PCR: Detection of the four families of OXA-type carbapenamases found in A. baumannii (bla OXA-23 like, bla $_{\text {OXA-40 }}$ like, bla $a_{\text {OXA-51 }}$ like and bla OXA-58 like) was done by a multiplex PCR. Extraction of DNA was done by using The GeneJET Genomic DNA Purification Kit (Thermo Scientific, Lithuania). PCR amplification of the four bla $a_{O X A}$ genes was performed with primers which were selected from published sequences (Karmostaji et al., 2013) and the four primer pairs were as

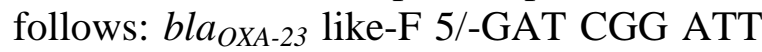
GGA GAA CCA GA-3// bla OXA-23 like-R 5/ATT TCT GAC CGC ATT TCC AT-3/;

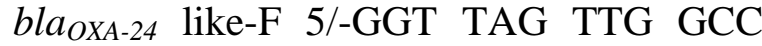
CCC TTA AA-3// bla OXA-24 like-R 5/-AGT
TGA GCG AAA AGG GGA TT-3/; bla $_{O X A-}$ ${ }_{51}$ like-F 5/-TAA TGC TTT GAT CGG CCT TG-3// bla OXA-51 like-R 5/-TGG ATT GCA CTT CAT CTT GG-3/; bla OXA-58 like-F 5/AAG TAT TGG GGC TTG TGC TG-3// bla ${ }_{O X A-58}$ like-R 5/-CCC CTC TGC GCT CTA CAT AC-3/ (Bioneer, Korea). PCR products [501 base pair (bp) (bla OXA-23 like), 246 bp (bla $a_{\text {OXA }-40}$ like), 353 bp (bla OXA-51 like) and 599 bp (bla OXA-58 like)] were visualized by agarose gel electrophoresis.

Sequencing of bla ${ }_{O X A-51}$ like gene: Further sequencing of bla $a_{O X A-51}$ like gene was done to $20 \mathrm{~A}$. baumannii isolates chosen from ICU infected cases. Sequencing was performed using an Applied Biosystems 3730XL sequencer at Macrogen Inc. (Korea). All sequences were identified using Basic Local Alignment Search Tool (BLAST) program. Homologous sequences to the sequence of interest were identified by a BLAST search using the sequence of interest as a query (Hall, 2013). According to GeneBank nucleotide database, 15 isolates were previously detected in gene bank while 5 isolates showed no similar results to those previously recorded. Multiple sequences alignment was accomplished using the Clustalw and the MUSCLE programs (Hall, 2013). Phylogenetic tree was constructed to determine molecular relatedness between isolates.

Statistical analysis: Data was analyzed using IBM SPSS advanced statistics version 20 (SPSS Inc., Chicago, IL, USA). Numerical data were expressed as mean and standard deviation or median and range as appropriate. Qualitative data were expressed as frequency and percentage. Chi-square test or Fisher's exact test was used to examine the relation between qualitative variables. Infection related mortality was analyzed using Kaplan-Meier method for survival 
analysis and comparison between two survival curves was done using log-rank test. All tests were two-tailed. A $p$-value < 0.05 was considered significant.

\section{Results and Discussion}

During one and a half year, MDR-AB were isolated from 82 non-duplicate specimens out of 544 gram negative organisms $(15.1 \%)$ growing from cultures of different clinical specimens. The demographic data and clinical characteristics of infected patients are summarized in table 1 . The mean age of patients was $46.3 \pm 17$ years. All isolates were identified as $A$. baumannii by the Microscan. The most common sites of infections by Acinetobacter pathogens were respiratory tract specimens accounting for $47.6 \% \quad(n=39)$ followed by surgical site infections $(n=36,43.9 \%)$, blood $(n=5,6.1 \%)$ and urine $(\mathrm{n}=2,2.4 \%)$.

Antimicrobial susceptibility profile of Acinetobacter isolates revealed sensitivity of $31(37.8 \%)$ to tobramycin, $25(30.5 \%)$ to gentamicin, $18(22.0 \%)$ to amikacin, 8 $(9.8 \%)$ to ciprofloxacin and $7(8.5 \%)$ to levofloxacin. Seventy eight isolates $(95.1 \%)$ were carbapenem resistant. The MBL phenotype was detected by CDST-IPM test in 66 isolates $(80.5 \%)$, in both imipenem sensitive and resistant isolates (Table 2). By multiplex PCR (Figure 1), bla OXA-51 like was detected in 77 isolates $(93.9 \%)$, while bla $_{\text {OXA-23 like was detected in } 52(63.4 \%) \text { of }}$ isolates and they coexisted in $62.2 \%$ of isolates. None of the isolates were bla $a_{\text {OXA-40 }}$ and bla $a_{\text {OXA-58 }}$ like genes positive. Four isolates $(4.9 \%)$ were negative for the four genes tested. The bla $a_{O X A}$ and MBL genes were simultaneously detected. The CDST positive results were associated with a positive bla $_{O X A-51}$ and bla ${ }_{O X A-23}$ like genes in $97 \%$ and $71 \%$ of isolates $(p=0.049,0.003$; respectively). However, $6.9 \%$ of imipenem resistant Acinetobacter isolates (5/72) were non MBL and non bla $a_{\text {OXA-23 }}$ carbapenemase producers. The phylogenetic analysis showed presence of several different genetic variants of $A$. baumannii isolates in our hospital (Figure 2).

Of the 82 patients infected by Acinetobacter pathogens, $44(53.7 \%)$ received empirical carbapenems (imipenem or meropenem), i.e. before documented proof of infection. Carbapenem therapy was significantly associated with OXA genes positivity as summarized in Table 3. The overall Acinetobacter infection related 14-day mortality was $32.5 \%$. The unadjusted (crude) 14-day mortality rates were significantly higher in patients who were admitted to ICU than in patients who were not admitted to ICU (52.4\% versus $12.4 \%$, $p<0.001)$. While 14-day mortality rates were not different between MBL, bla $a_{O X A}$ genes (bla $a_{O X A} 51$ and bla ${ }_{O X A}{ }_{23}$ ) positive and negative cases $(p=0.686, \quad p=0.453$ and $p=0.779$; respectively).

In the present study, Acinetobacter constituted $15.1 \%$ of gram negative pathogens isolated from different clinical specimens of adult cancer patients. Similar figures were previously reported in cancer patients (18.7\%) (Eldomany and Abdelaziz, 2011).

The increased rate of antimicrobial resistant Acinetobacter, specifically to carbapenems, constitute a problem in the management of the infections caused by these pathogens. In the current study, a high rate of carbapenem resistance has been recorded among MDR Acinetobacter isolates (95.1\%); more pronounced against meropenem than imipenem (93.9\% and 87.8\%; respectively). The presence of efflux pump over expression as a resistance mechanism that pumps out meropenem but not imipenem is 
a possible explanation for more meropenem resistance (Meletis et al., 2012).

In the current study, A. baumannii infections were mostly health-care acquired (98.8\%), and were significantly associated with prolonged hospitalization as $76.8 \%$ of patients spent $\geq 7$ days in hospital prior to developing infection. Similarly, Dash et al. reported that the majority of $A$. baumannii infections were detected from inpatients (90.5\%) with $75.9 \%$ of cases spending 7 or more days in the hospital (Dash et al., 2013). Vaze and his colleagues recorded that $71 \%$ of patients infected by A. baumannii were hospitalized for more than one week (Vaze et al., 2013). Sixty two percent of cases were subjected to surgical procedures one month prior to infection; pointing to the importance of invasive procedures to acquisition of infection. In addition, $48.8 \%$ of A. baumannii infections were isolated from patients admitted to ICU. The importance of Acinetobacter species as a cause of ICU infections was previously confirmed (Kim et al., 2012).

The MBL phenotype was detected in $80.5 \%$ of Acinetobacter isolates in the present study; in both imipenem susceptible and imipenem resistant isolates. Similar results were reported in another Egyptian study, as MBLs were positive in $86.7 \%$ of imipenem resistant $A$. baumannii (IRAB) by CDST (Fattouh and Nasr El-din, 2014). Other studies demonstrated MBLs positivity in $50 \%$ of IRAB isolates by CDST (Hodiwala et al., 2013; Mohamed and Rafaat, 2011). The detection of MBLs among imipenem sensitive isolates was reported in some studies with rates up to $20 \%$ (Daef et al., 2012). Thus, it is evident that MBLs are detected among imipenem resistant isolates, as well as imipenem susceptible ones. The carbapenem susceptible pathogens with hidden MBL genes might disseminate resistance in health-care facilities if not screened for the presence of MBLs.

The bla $a_{O X A-51}$ and bla $a_{O X A-23}$ genes were positive in $93.9 \%$ and $63.4 \%$, of $A$. baumannii pathogens in the current study, respectively. However, bla $a_{O X A-40}$ and bla OXA- $_{\text {- }}$ 58 genes were not detected in any isolate. In a similar study, bla $a_{O X A-51}$ like, bla $a_{O X A-23}$ like, bla $a_{\text {OXA-40 }}, b_{\text {OXA-58 }}$ like were positive in $100 \%, 50 \%, 7.5 \%$, and $5 \%$ of IRAB isolates, from three different hospitals in Egypt (AlAgamy et al., 2014). Also bla OXA-51 like, bla $a_{\text {OXA-23 like, bla }}$ OXA-58 like and bla OXA-40 like were detected in $100.0 \%, 55.9 \%, 14.7 \%$ and $2.9 \%$, respectively of $A$. baumannii isolates from pediatric patients in Egypt (AlHassan et al., 2013). However, bla OXA-5l like and the bla OXA-23 $_{2}$ like genes were reported in all of the CRAB isolates from ICUs in 3 hospitals including NCI, in Egypt (Fouad et al., 2013).

Similarly, bla $a_{\text {OXA-23 like genes were detected }}$ in $60.0 \%$ and $100.0 \%$ of IRAB from different parts of the world, and bla $a_{O X A-51}$ like was positive in all strains; whereas bla $a_{O X A} 40$ like and bla $a_{O X-58}$ like genes were not detected in many studies (Prata-Rocha et al., 2013).

Many studies confirmed the presence of multiple genes encoding carbapenem hydrolyzing enzymes in $A$. baumannii (Fattouh and Nasr El-din, 2014). In the present study, positive CDST Acinetobacter isolates were significantly associated with positivity of the bla $a_{O X A-51}(97.0 \%)$ and the bla $_{\text {OXA-23 }}$ like genes $(71.2 \%) ; p=0.049$, 0.003 ; respectively. This was previously confirmed as $80.6 \%$ of $A$. baumannii strains with positive results in CDST were positive for the bla OXA-23 like genes (Azimi et al., 2013). Still, other mechanisms of carbapenem resistance such as modification of penicillin binding proteins, loss of porins 
and/or altered efflux pump activity should be investigated as $6.9 \%$ of IRAB were non MBL and non bla $a_{O X-23}$ carbapenemase producers.

The growing rate of carbapenem resistance in the current study, could be partially explained by the widespread clinical use of these antibiotics in our hospital, as nearly half $(53.7 \%)$ of our patients received carbapenem therapy prior to documenting infection. In addition, carbapenem therapy was significantly associated with OXA genes positivity $(100 \%$ and $75 \%$ for bla OXA51 and bla ${ }_{O X A-23}$ with a $p$-value 0.015 and 0.064 ; respectively. It is evident that exposure to antibiotics effectively selects the MDR strains and allow the acquisition of more antibiotic resistance genes.

Table.1 Demographic data and clinical characteristics

\begin{tabular}{|c|c|c|}
\hline Parameter & Total number & Percentage $(\%)$ \\
\hline \multicolumn{3}{|l|}{ Age } \\
\hline $18-40$ years & 31 & 37.8 \\
\hline $41-64$ years & 35 & 42.7 \\
\hline$\geq 65$ years & 16 & 19.5 \\
\hline \multicolumn{3}{|l|}{ Gender } \\
\hline Male & 35 & 42.7 \\
\hline Female & 47 & 57.3 \\
\hline \multicolumn{3}{|l|}{ Department } \\
\hline Surgical & 57 & 69.5 \\
\hline Medical & 25 & 30.5 \\
\hline \multicolumn{3}{|l|}{ ICU admission } \\
\hline No & 42 & 51.2 \\
\hline Yes & 40 & 48.8 \\
\hline \multicolumn{3}{|l|}{ Prior hospitalization } \\
\hline No & 60 & 73.2 \\
\hline Yes & 22 & 26.8 \\
\hline \multicolumn{3}{|l|}{ Hospitalization } \\
\hline Inpatient & 81 & 98.8 \\
\hline Outpatient & 1 & 1.2 \\
\hline \multicolumn{3}{|l|}{ Duration of hospital stay before infection } \\
\hline$<7$ days & 19 & 23.2 \\
\hline 7 days $-<30$ days & 47 & 57.3 \\
\hline$\geq 30$ days & 16 & 19.5 \\
\hline \multicolumn{3}{|l|}{ Underlying disease } \\
\hline Hematological tumor & 24 & 29.3 \\
\hline Solid tumor & 58 & 70.7 \\
\hline \multicolumn{3}{|l|}{ Surgery within 1 month } \\
\hline No & 31 & 37.8 \\
\hline Yes & 51 & 62.2 \\
\hline \multicolumn{3}{|l|}{ Chemotherapy within 1 month } \\
\hline No & 59 & 72.0 \\
\hline Yes & 23 & 28.0 \\
\hline
\end{tabular}


Table.2 Relation of carbapenem susceptibility to MBL production and blaoxa genes positivity

\begin{tabular}{|c|c|c|c|c|c|c|}
\hline & Imipener & $($ No. $(\%))$ & & Meropen & n (No. (\%)) & \\
\hline & $\begin{array}{l}\text { Sensitive } \\
(n=10)\end{array}$ & $\begin{array}{c}\text { Resistance } \\
(\mathrm{n}=72)\end{array}$ & $P$-value & $\begin{array}{c}\text { Sensitive } \\
\quad(n=5)\end{array}$ & $\begin{array}{c}\text { Resistance } \\
\quad(n=77)\end{array}$ & $P$-value \\
\hline CDST-IPM test & & & & & & \\
\hline Negative $(n=16)$ & $6(60.0)$ & $10(13.9)$ & & $3(60.0)$ & $13(16.9)$ & \\
\hline Positive $(\mathrm{n}=66)$ & $4(40.0)$ & $62(86.1)$ & 0.001 & $2(40.0)$ & $64(83.1)$ & 0.049 \\
\hline bla $_{O X A} 51$ & & & & & & \\
\hline Negative $(n=5)$ & $3(30.0)$ & $2(2.8)$ & & $2(40.0)$ & $3(3.9)$ & \\
\hline Positive $(n=77)$ & $7(70.0)$ & $70(97.2)$ & 0.012 & $3(60.0)$ & $74(96.1)$ & $*$ \\
\hline bla $_{O X A 23}$ & & & & & & \\
\hline Negative $(n=30)$ & $10(100.0)$ & $20(27.8)$ & & $4(80.0)$ & $26(33.8)$ & \\
\hline Positive $(n=52)$ & $0(0.0)$ & $52(72.2)$ & $<0.001$ & $1(20.0)$ & $51(66.2)$ & 0.057 \\
\hline
\end{tabular}

Table.3 Empirical Carbapenem use in relation to multiplex PCR results for the blaoxa genes

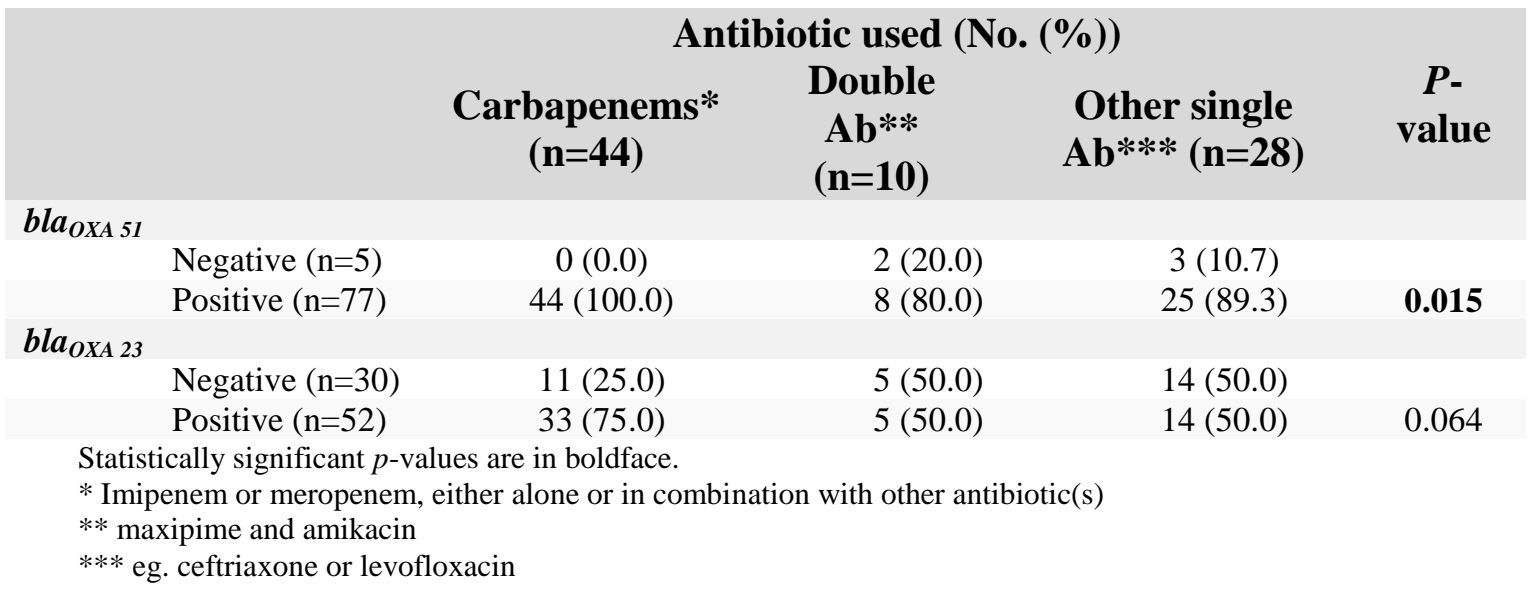

Fig.1 Detection of genes encoding OXA carbapenamases by multiplex PCR. Lanes 1, 4, 6, 8, 9,18 blaOXA-51 like gene; Lanes 2, 3, 5, 7, 10-17 blaOXA-23 like and blaOXA-51 like genes; DNA ladder marker (50 bp DNA ladder)

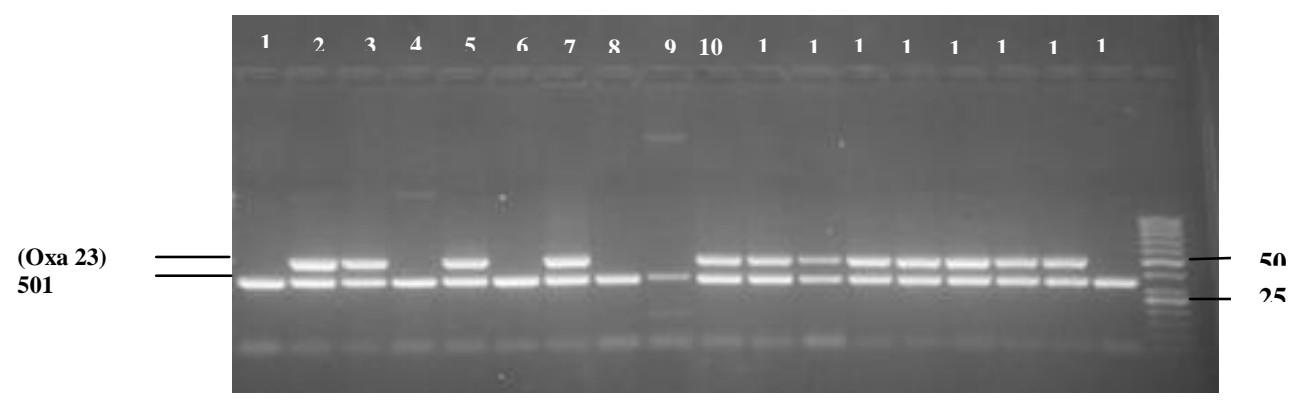


Fig.2 Molecular Phylogenetic analysis by Maximum Likelihood method based on sequencing blaOXA-51 gene from different 15 isolates of A. baumannii. The dendrogram shows the amounts of genetic change based on multiple alignments and were generated using MEGA5 software. The bar at the bottom of the figure shows the amount of genetic change corresponding to the length of each branch

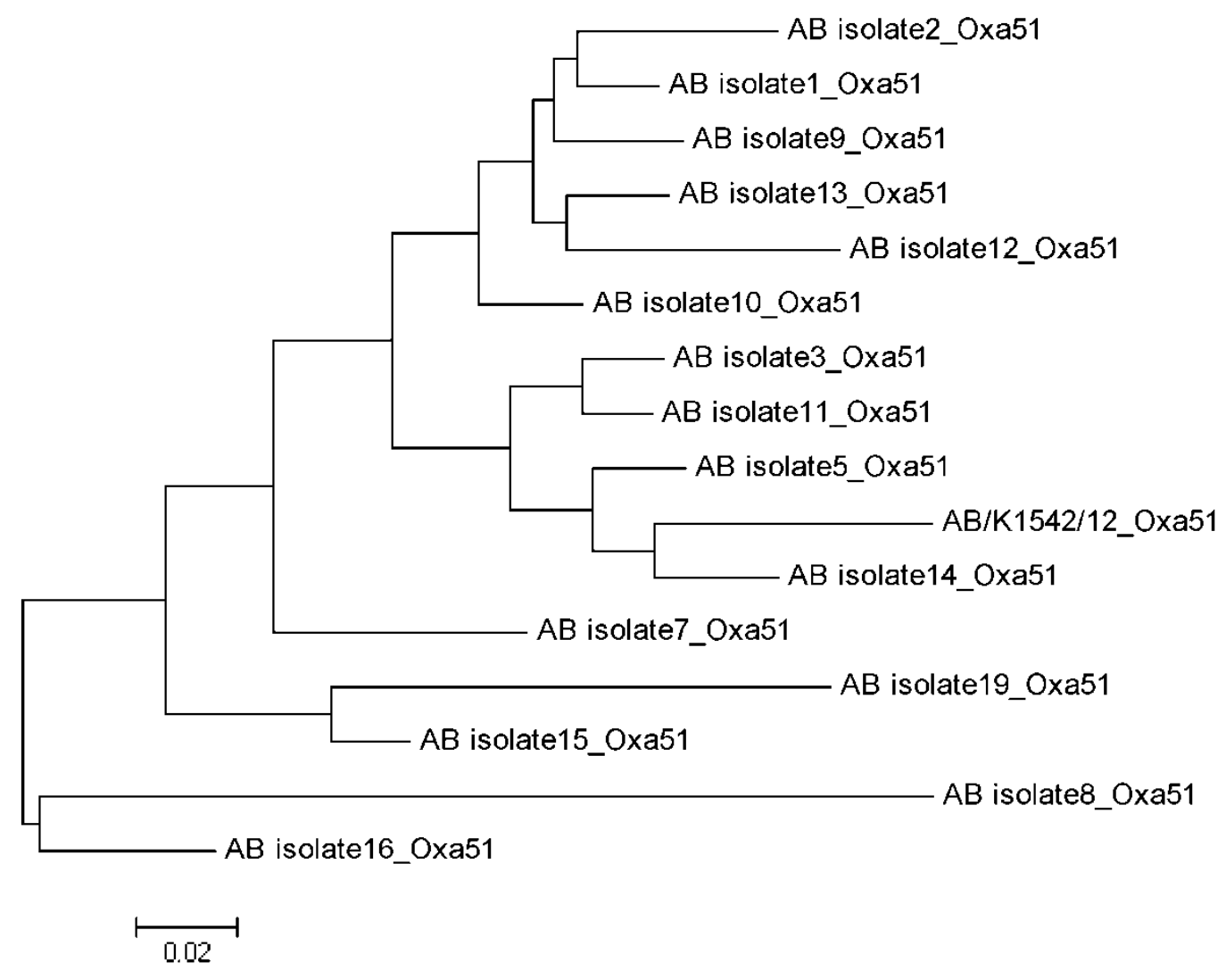

A phylogenetic tree was originally used in the assessment of the relation between DNA sequences and their hypothetical common ancestors; mainly among the species represented by those sequences. Nowadays it is used to understand the relationships among the sequences regardless of their host species, estimating the functions of genes that have not been studied experimentally, and helping in investigating microbial outbreaks (Hall, 2013). In the current study, a large diversity was found in the sequences of bla $O X A-51$ like gene. This diversity suggested sporadic cases of $A$. baumannii rather than the presence of a single clone. This was against expectations, given the short duration of sample collection (1.5 years) and the fact that isolates studied were those recovered from ICU. This could be due to transfer of cases from different wards in the hospital to the ICU. The plasticity of Acinetobacter genetic composition allow easy inclusion of new genes, thus making it difficult to define its origin (Al-Hassan et al., 2013). Similarly, in a previous study done on Egyptian pediatric cancer patients, diversity of $A$. baumannii isolates was confirmed (Al-Hassan et al., 2013).

The most common site of infection associated with Acinetobacter pathogens in the present study, was hospital acquired pneumonia; as respiratory tract specimens accounted for almost half of isolates 
(47.6\%). This was previously documented as $A$. baumannii were isolated primarily from respiratory tract specimens (49\%) (Vaze et al., 2013), and endotracheal secretions (33.3\%) (Fattouh and Nasr El-din, 2014). In general, these opportunistic pathogens are more commonly isolated from high risk hospitalized patients subjected to invasive procedures. Thus, increased rates of MDR-AB infections and its diversity might be due to the presence of several risk factors in our patients like ICU admission, surgical procedures and prolonged hospitalization.

A high crude 14-day mortality rate was associated with infections caused by MDR A. baumannii infections in the present study $(32.5 \%)$. Similar high 14-day mortality rates were previously recorded with Acinetobacter infections (Kim et al., 2012). Still the exact cause of mortality is not clear as these infections usually occur in high risk ICU patients with high rates of comorbidity. Therefore, further studies are needed to focus on the possible multifactorial parameters contributing for the unfavorable outcome.

Thus, we concluded that MDR Acinetobacter infections constituted important causes of HAI in cancer patients. These infections were significantly associated with prolonged hospital stay and prior intake of carbapenems. It is evident that multiple mechanisms of resistance contributed to the increasing rates of carbapenem resistance in A. baumannii. Diversity was encountered in A. baumannii isolates. The diversity of isolates and multiple mechanisms of carbapenem resistance suggested that acquisition of MDR-AB infections might be a multistep complex process that took place as a result of multiple risk factors, including invasive procedures, carbapenem therapy and prolonged duration of hospitalization; rather than simple health-care associated transmission.

\section{Funding}

This study was done as a part of NCI work and was funded by NCI.

\section{Conflict of Interest}

No conflict of interest is considered for any of the authors of this study.

\section{References}

Al-Agamy, M., H., Khalaf, N., G., Tawfick, M., M., Shibl, A., M., and El Kholy, A. 2014. Molecular characterization of carbapenem insensitive Acinetobacter baumannii in Egypt. Int. J. Infect. Dis., 22: 49-54.

Al-Hassan, L., El Mehallawy, H., and Amyes, S.G.B. 2013. Diversity in Acinetobacter baumannii isolates from paediatric cancer patients in Egypt. Clin. Microbiol. Infect. Dis., 19: 10821088.

Azimi, L., Lari, A., R., Talebi, M., Namvar, A., E., and Jabbari, M. 2013. Comparison between phenotypic and PCR for detection of OXA-23 type and metallo-beta-lactamases producer Acinetobacter spp. GMS Hygiene and Infection Control, 8, 1-6.

CLSI. 2010. Performance standards for antimicrobial susceptibility testing; twentieth informational supplement. CLSI document M100-S20. Clinical and Laboratory Standards Institute, Wayne, PA.

Daef, E., A., Mohamed, I., S., Ahmed, A., S., Elsherbiny, N., M., and Sayed, I., M. 2012. Evaluation of different phenotypic assays for the detection of metallo- $\beta$-lactamase production in carbapenem susceptible and resistant 
Acinetobacter baumannii isolates. $J$. American Sci., 8, 292-299.

Dash, M., Padhi, S., Pattnaik, S., Mohanty, I., and Misra, P. 2013. Frequency, risk factors, and antibiogram of Acinetobacter species isolated from various clinical samples in a tertiary care hospital in Odisha, India. Avicenna J. Med., 3, 97-102.

Eldomany, R., and Abdelaziz, N., A. 2011. Characterization and antimicrobial susceptibility of gram negative bacteria isolated from cancer patients on chemotherapy in Egypt. Arch. Clin. Microbiol., 2: 1-13.

Fattouh, M., and Nasr El-din, A. 2014. Emergence of carbapenem-resistant acinetobacter baumannii in the intensive care unit in Sohag University Hospital, Egypt. Int. J. Curr. Microbiol. Appl. Sci., 3, 732-744.

Fouad, M., Attia, A., S., Tawakkol, W., M., and Hashem, A., M. 2013. Emergence of carbapenem resistant Acinetobacter baumannii harboring the OXA-23 carbapenemase in intensive care units of Egyptian hospitals. Int. J. Infect. Dis., 17, e1252-e1254.

Hall, B., G. 2013. Building Phylogenetic Trees from Molecular Data with MEGA. Mol. Biol. Evol., 30: 12291235.

Hodiwala, A., Dhoke, R., and Urhekar, A., D. 2013. Incidence of metallo-betalactamase producing pseudomonas, acinetobacter \& enterobacterial isolates in hospitalised patients. Int. J. Pharm. Biol. Sci., 3: 79-83.

Karmostaji, A., Peerayeh, S., N., and Salmanian, A., H. 2013. Distribution of OXA-type class D $\beta$-lactamase genes among nosocomial multi drug resistant Acinetobacter baumannii isolated in Tehran hospitals. Jundishapur J. Microbiol., 6, e8219e8223.
Kim, Y., J., Kim, S., Hong, K., W., Kim, Y., R., Park, Y., J., and Kang, M., W. 2012. Risk Factors for Mortality in Patients with Carbapenem-Resistant Acinetobacter baumannii Bacteremia: Impact of Appropriate Antimicrobial Therapy. J. Korean Med. Sci., 27: 471475.

Lee, M., H., Chen, T., L., Lee, Y., T., Huang, L., Kuo, S., C., Yu, K., W., Hsueh, P., R., Dou, H., Y., Su, I., J., and Fung, C., P. 2013. Dissemination of multidrug resistant Acinetobacter baumannii carrying bla ${ }_{O X A-23}$ from hospitals in central Taiwan. $J$. Microbiol. Immunol. Infect., 46, 419. 424.

Liao, Y., T., Kuo, S., C., Lee, Y., T., Chen, C., P., Lin, S., W., Shen, L., J., Fung, C., P., Cho, W., L., and Chen, T., L. 2014. Sheltering effect and indirect pathogenesis of carbapenem-resistant Acinetobacter baumannii in polymicrobial infection. Antimicrobial Agents and Chemotherapy. 58, 39833990.

Meletis, G., Exindari, M., Vavatsi, N., Sofianou, D., and Diza, E. 2012. Mechanisms responsible for the emergence of carbapenem resistance in Pseudomonas aeruginosa. Hippokratia, 16, 303-307.

Mohamed, N., M., and Raafat, D. 2011. Phenotypic and genotypic detection of metallo beta lactamases in imipenem resistant Acinetobacter baumannii isolated from a tertiary hospital in Alexandria, Egypt. Res. J. Microbiol., $6,750-760$.

Opazo, A., Domínguez, M., Bello, H., Amyes, S., G., B., and GonzálezRocha, G. 2012. OXA-type carbapenemases in Acinetobacter baumannii in South America. $J$. Infection in Developing Countries, 6, 311-316. 
Prata-Rocha, M., L., Moreira, M., R., Gontijo Filho, P., P., and Melo, G., B. 2013. Acinetobacter baumannii: global evolution of carbapenemresistant and genotyping methods. Microbial pathogens and strategies for combating them. science, technology and education. 1, 197-203.
Vaze, N., D., Emery, C., L., Hamilton, R., J., Brooks, A., D., and Joshi, S., G. 2013. Patient demographics and characteristics of infection with carbapenem resistant acinetobacter baumannii in a teaching hospital from the United States. Adv. Infect. Dis., 3, 10-16.

\section{How to cite this article:}

Rasha M. Abdel Hamid, Safaa S. Hassan, Hadir A. El-Mahallawy and Magdy Saber. 2016. Molecular Characterization of Carbapenem Resistant Acinetobacter baumannii in Cancer Patients. Int.J.Curr.Microbiol.App.Sci. 5(11): 637-647. doi: http://dx.doi.org/10.20546/ijcmas.2016.511.075 